\title{
Semidefinite programming based tests for matrix copositivity
}

\author{
Pablo A. Parrilo \\ pablo@cds.caltech.edu \\ Control \& Dynamical Systems 107-81 \\ California Institute of Technology \\ Pasadena, CA 91125-8100
}

\begin{abstract}
The verification of matrix copositivity is a well known computationally hard problem, with many applications in continuous and combinatorial optimization. In this paper, we present a hierarchy of semidefinite programming based sufficient conditions for a real matrix to be copositive. These conditions are obtained through the use of a sum of squares decomposition for multivariable forms. As can be expected, there is a tradeoff between conservativeness of the tests and the corresponding computational requirements. The proposed tests are shown to be exact for a certain family of extreme copositive matrices.
\end{abstract}

\section{Introduction}

A real matrix $M$ is said to be copositive if the quadratic form $x^{T} M x$ takes only positive values in the nonnegative orthant. As opposed to positive definiteness, which can be efficiently verified (for example, using the Cholesky decomposition), there are no polynomial time algorithms for checking copositiveness, unless $\mathrm{co}-\mathrm{NP}=\mathrm{NP}$.

Copositive matrices have numerous applications in diverse fields of applied mathematics, especially in optimization. It is a critical ingredient in the characterization of local solutions of constrained optimization problems [10], such as the linear complementarity problem [3]. Also, it has been recently shown that its use can notably improve certain convex relaxation bounds in quadratic programming problems with linear constraints [15]. These relaxations are the underlying basis of many important results in robustness analysis. A recent example of its application in a control setting is in the stability analysis using piecewise quadratic Lyapunov functions [8].

From a computational complexity viewpoint, the recognition problem for copositive matrices is hard, in general. It has been shown that checking if a given ma- trix is not copositive is an NP-complete problem [10]. Equivalently, checking copositivity is in co-NPC (see $[5,11]$ for background material on computational complexity). This implies that, unless co- $\mathrm{NP}=\mathrm{NP}$ (a consequence of $\mathrm{P}=\mathrm{NP}$ ), in general it is not possible to construct polynomial time certificates of copositivity (i.e., copositivity is not in NP).

In many cases, however, it is possible to efficiently construct such certificates. For example, assume that the matrix $M$ has a decomposition $M=P+N$, with $P$ positive semidefinite and $N$ componentwise nonnegative. It is clear that this implies that $M$ is copositive, with the matrices $P$ and $N$ providing a polynomial time verifiable certificate.

In a similar way, the results in this paper provide a unified methodology of constructing sufficient conditions for copositivity. The procedure uses as a basic tool a sum of squares decomposition for multivariable forms, which can be obtained using semidefinite programming methods $[19,14,13]$. One of the main advantages of the proposed procedure is that it can also be applied to the case when the coefficients of $M$ are variable (or uncertain).

The structure of the paper is as follows: in section 2 we introduce the notation and some background material. In section 3 a procedure to complute a sum of squares decomposition for a multivariable form is reviewed. In the next section, the SOS decomposition and Pólya's theorem on positive definite forms are employed to produce copositivity certificates. In section 5 we present numerical examples, and finally, future research directions are outlined and conclusions are made.

\section{Background}

The notation is mostly standard. A matrix $M \in \mathbb{R}^{n \times n}$ is copositive if $x^{T} M x \geq 0 \quad \forall x \in \mathbb{R}^{n}, x_{i} \geq 0$. Equivalently, the quadratic form is nonnegative on the closed nonnegative orthant. If $x^{T} M x$ takes only positive values on the closed orthant (except the origin, of course), 
A set $S \subseteq \mathbb{R}^{n}$ is a said to be a cone if $\lambda \geq 0, x \in$ $S \Rightarrow \lambda x \in S$. A set $S$ is convex if $x_{1}, x_{2} \in S$ implies $\lambda x_{1}+(1-\lambda) x_{2} \in S$ for all $0 \leq \lambda \leq 1$. The dual of a set $S$ is $S^{*}=\left\{y \in \mathbb{R}^{n}: x \in S \Rightarrow\langle x, y\rangle \geq 0\right\}$. A point $x$ of a convex cone $C$ is an extreme point if $x=x_{1}+x_{2}$, $x_{i} \in C$ implies $x_{1}=\lambda x, x_{2}=(1-\lambda) x, 0 \leq \lambda \leq 1$. It can be shown that the set of copositive matrices $\mathcal{C}$ is a closed convex cone [6]. We also denote as $\mathcal{P}, \mathcal{N}$ the self-dual cones of positive semidefinite and elementwise nonnegative matrices, respectively.

Denote by $\mathcal{F}_{n, m}$ the set of homogeneous forms of degree $m$ in $n$ variables $\left\{x_{1}, \ldots, x_{n}\right\}$, with real coefficients. Every such form can be written as a sum of $\left(\begin{array}{c}n+m-1 \\ m\end{array}\right)$ monomials, each one of the form $c_{\alpha} \prod_{i=1}^{n} x_{i}^{\alpha_{i}}$, with $\sum_{i=1}^{n} \alpha_{i}=m$. A form $F \in \mathcal{F}_{n, m}$ is nonnegative (or positive semidefinite) if $F(x) \geq 0$ for all $x \in \mathbb{R}^{n}$. It is positive definite if $F(x)>0$ for all $x \in \mathbb{R}^{n}, x \neq 0$.

There exist in the literature explicit necessary and sufficient conditions for a given matrix to be copositive. These conditions are usually expressed in terms of principal minors (see $[21,3]$ and the references therein). However, since checking copositivity of a matrix is a co-NP-complete problem [10], this implies that in the worst case these tests can take an exponential number of operations (unless $P=N P$ ). Thus, the need for efficient sufficient conditions to guarantee copositivity.

We briefly describe two applications of copositive matrices, mentioned in the introduction. Consider first the problem of obtaining a lower bound on the optimal solution of a linearly constrained quadratic optimization problem [15]:

$$
f^{*}=\min _{A x \geq 0, x^{T} x=1} x^{T} Q x
$$

If there exists a solution of the LMI

$$
Q-A^{T} C A \geq \gamma I
$$

where $C$ is a copositive matrix, then it immediately follows that $f^{*} \geq \gamma$. Thus, having semidefinite programming tests for copositivity allows for enhanced bounds for this type of problems. The other application, presented in [8], deals with the analysis of piecewise linear systems using piecewise quadratic Lyapunov functions. One of the basic issues in that problem is checking nonnegativity of the Lyapunov function, in a region (or "cell") defined by linear inequalities. To this end, an LMI-based sufficient condition is usually employed. By using the improved copositivity tests presented in this paper, less conservative answers can be obtained, especially in the case of systems of high state dimension. The conditions in [8] basically correspond to the suffcient condition (3).
Let $F(x) \in \mathcal{F}_{n, m}$ be a homogeneous form of degree $m$ in $n$ variables $\left\{x_{1}, \ldots, x_{n}\right\}$, with real coefficients. A sufficient condition for $F$ to be nonnegative is the existence of a sum of squares decomposition $F(x)=\sum_{i} f_{i}^{2}(x)$ (of course, $m$ has to be even). If $F$ is nonnegative, and the $f_{i}(x)$ are constrained to be rational functions, the existence of this decomposition is guaranteed by Artin's solution to Hilbert's 17th problem. If the functions $f_{i}(x)$ should instead be homogeneous forms, then the existence is guaranteed only in certain cases, depending on the values of $n$ and $m$ [16]. It has been shown $[19,14,13]$ that this decomposition, if it exists, can be obtained by using semidefinite programming methods. We briefly outline the methodology below; a complete description can be found in the references mentioned earlier.

The basic idea of the method is the following: express the given form $F(x)$ as a quadratic form in some new variables $z$. These new variables are all the monomials of degree equal to $\frac{1}{2} m$ given by the different products of the $x$ variables. Therefore, $F(x)$ can be represented as

$$
F(x)=z^{T} Q z
$$

where $Q$ is a constant matrix. If in the representation above $Q$ is positive semidefinite, then $F(x)$ takes only positive values. In principle, this condition is conservative, generally speaking. The main reason is that since the variables $z_{i}$ are not independent the representation (1) might not be unique, and $Q$ may be positive semidefinite for some representations, but not for others. Fortunately, this conservativeness can be avoided by using identically satisfied constraints that relate the $z_{i}$ variables among themselves (of the form $z_{i} z_{j}=z_{k} z_{l}$ or $z_{i}^{2}=z_{k} z_{l}$ ). This way, it is easily shown that there is a linear subspace of matrices $Q$ that satisfy (1). If the intersection of this subspace with the positive semidefinite matrix cone is nonempty (which can be checked by solving an LMI feasibility problem), then the original function $F$ is guaranteed to be a sum of squares, and therefore positive semidefinite. This follows from a decomposition of $Q=L^{T} L$, which implies the sum of squares representation $F(x)=\sum_{i}(L z)_{i}^{2}$. Conversely, if $F$ can indeed be written as the sum of squares of forms, then expanding in monomials will provide the representation (1).

Example 1 Consider the following quartic form in two variables:

$$
\begin{aligned}
F\left(x_{1}, x_{2}\right) & =2 x_{1}^{4}+2 x_{1}^{3} x_{2}-x_{1}^{2} x_{2}^{2}+5 x_{2}^{4} \\
& =\left[\begin{array}{c}
x_{1}^{2} \\
x_{2}^{2} \\
x_{1} x_{2}
\end{array}\right]^{T}\left[\begin{array}{ccc}
2 & 0 & 1 \\
0 & 5 & 0 \\
1 & 0 & -1
\end{array}\right]\left[\begin{array}{c}
x_{1}^{2} \\
x_{2}^{2} \\
x_{1} x_{2}
\end{array}\right] \\
& =\left[\begin{array}{c}
x_{1}^{2} \\
x_{2}^{2} \\
x_{1} x_{2}
\end{array}\right]^{T}\left[\begin{array}{ccc}
2 & -\lambda & 1 \\
-\lambda & 5 & 0 \\
1 & 0 & -1+2 \lambda
\end{array}\right]\left[\begin{array}{c}
x_{1}^{2} \\
x_{2}^{2} \\
x_{1} x_{2}
\end{array}\right]
\end{aligned}
$$


Taking for instance $\lambda=3$, the matrix in the last expression is positive semidefinite. In this case,

$$
Q=L^{T} L, \quad L=\frac{1}{\sqrt{2}}\left[\begin{array}{ccc}
2 & -3 & 1 \\
0 & 1 & 3
\end{array}\right]
$$

and therefore we obtain the sum of squares decomposition:

$$
F\left(x_{1}, x_{2}\right)=\frac{1}{2}\left(\left(2 x_{1}^{2}-3 x_{2}^{2}+x_{1} x_{2}\right)^{2}+\left(x_{2}^{2}+3 x_{1} x_{2}\right)^{2}\right) .
$$

\section{Main Results}

In order to apply the sum of squares decomposition to the matrix copositivity problem, we need a way of dealing with the constraints in the variables, since each $x_{i}$ has to be nonnegative. A natural way of addressing this issue is the following: to check copositivity of $M$, we can consider $x_{i}=z_{i}^{2}$ and study the global nonnegativity of the fourth order form given by:

$$
P(\mathbf{z}):=\mathbf{x}^{T} M \mathbf{x}=\sum_{i, j} m_{i j} z_{i}^{2} z_{j}^{2}
$$

It is easy to verify that $M$ is copositive if and only if the form $P(\mathbf{z})$ is positive semidefinite. Therefore, an obvious sufficient condition for $M$ to be copositive is that $P(\mathbf{z})$ can be written as a sum of squares.

In order to do that, as explained in the previous section, we have to express $P(z)$ as a quadratic form in the variables $z_{i}^{2}$ and $z_{i} z_{j}$, for $i \neq j$. In principle, the order of the new matrix $Q$ is now $n+\left(\begin{array}{l}n \\ 2\end{array}\right)$. The nonuniqueness of the representation follows from the identities

$$
\begin{aligned}
\left(z_{i} z_{j}\right)^{2} & =\left(z_{i}^{2}\right)\left(z_{j}^{2}\right) \\
\left(z_{i} z_{j}\right)\left(z_{i} z_{l}\right) & =\left(z_{i}^{2}\right)\left(z_{j} z_{l}\right) \\
\left(z_{i} z_{j}\right)\left(z_{k} z_{l}\right) & =\left(z_{i} z_{k}\right)\left(z_{j} z_{l}\right)=\left(z_{i} z_{l}\right)\left(z_{j} z_{k}\right) .
\end{aligned}
$$

Denote the associated free multipliers by the variables $\lambda_{i j}, \nu_{i j l}$, and $\mu_{i j k l}, \mu_{i j k l}^{\prime}$ respectively. By grouping the variables in a vector $\mathbf{Z}$ (first the $z_{i}^{2}$, then the $z_{i} z_{j}$ ), and writing

$$
P(\mathbf{z})=\mathbf{Z}^{T} Q \mathbf{Z},
$$

the matrix $Q$ can be shown to have the structure shown in Table 1, where the places with asterisks are either zero or a linear combination of the $\nu$ and $\mu$ variables.

Therefore, $P(\mathbf{z})$ will have a sum of squares decomposition if and only if there exists variables $\lambda, \mu, \nu$ such that the matrix $Q$ in Table 1 is positive semidefinite, i.e., if a certain LMI is feasible. Without loss of generality, it is always possible to choose the $\mu, \nu$ equal to zero without changing the feasibility of the LMI, since these terms appear only in the off-diagonal subblocks.
Consequently, all the $\lambda_{i j}$ should be nonnegative, and the LMI can be reduced to:

$$
\left.\begin{array}{cccc}
m_{11} & m_{12}-\lambda_{12} & \ldots & m_{1 n}-\lambda_{1 n} \\
m_{12}-\lambda_{12} & m_{22} & \ldots & m_{2 n}-\lambda_{2 n} \\
\vdots & \vdots & \ddots & \vdots \\
m_{1 n}-\lambda_{1 n} & m_{2 n}-\lambda_{2 n} & \ldots & m_{n n}
\end{array}\right] \geq 0
$$

It is easy to verify that existence of such $\lambda_{i j}$ turns out to be equivalent to the condition that the original matrix $M$ can be written as the sum of a positive semidefinite and an elementwise nonnegative matrix, i.e.,

$$
M=P+N, \quad P \geq 0, \quad n_{i j} \geq 0 .
$$

As mentioned in the introduction, this is a well-known sufficient condition for copositivity (see for example [4]). The equivalence between these two tests has also been noticed in [2, Lemma 3.5]. Note also that condition (3) can be obtained by considering the enhanced Shor relaxation, where new quadratic constraints are obtained by considering the pairwise products of linear constraints [15].

From what we have seen so far, we are able to obtain a sufficient test for copositivity, based on the sum of squares framework. The advantage of the approach in this paper is that even stronger conditions can be derived. By considering higher order forms, a hierarchy of increasingly powerful tests is obtained. Of course, the computational requirements increase accordingly.

Take for example the family of $2(r+2)$-forms given by

$$
P_{r}(\mathbf{z})=\left(\sum_{i=1}^{n} z_{i}^{2}\right)^{r} P(\mathbf{z})
$$

Then it is easy to see that if $P_{i}$ is a sum of squares, then $P_{i+1}$ is also a sum of squares. The converse proposition does not necessarily hold, i.e., $P_{i+1}$ can be a sum of squares, while $P_{i}$ is not. Additionally, if $P_{r}(\mathbf{z})$ is nonnegative, then so is $P(\mathbf{z})$. So, by testing if $P_{r}(\mathbf{z})$ is a sum of squares (which can be done using LMI methods, as described), we can guarantee the nonnegativity of $P(\mathbf{z})$, and as a consequence, copositivity of $M$.

For concreteness, we will analyze in some detail the case $r=1$. We will see that as in the case for $r=0$ described above, some variables automatically drop out from the optimization due to the particular structure of the resulting LMIs.

As explained, we consider now the sixth order form:

$$
P_{1}(\mathbf{z}):=\sum_{i, j, k} m_{j k} z_{i}^{2} z_{j}^{2} z_{k}^{2} .
$$




$$
Q=\left[\begin{array}{cccccccc}
m_{11} & m_{12}-\lambda_{12} & \ldots & m_{1 n}-\lambda_{1 n} & * & * & * & * \\
m_{12}-\lambda_{12} & m_{22} & \ldots & m_{2 n}-\lambda_{2 n} & * & * & * & * \\
\vdots & \vdots & \ddots & \vdots & \vdots & \vdots & \vdots & \vdots \\
m_{1 n}-\lambda_{1 n} & m_{2 n}-\lambda_{2 n} & \ldots & m_{n n} & * & * & \vdots & * \\
* & * & \ldots & * & 2 \lambda_{12} & * & \ldots & * \\
* & * & \ldots & * & * & 2 \lambda_{13} & \ldots & * \\
\vdots & \vdots & \vdots & \vdots & \vdots & \vdots & \ddots & \vdots \\
* & * & \ldots & * & * & * & \ldots & 2 \lambda_{(n-1) n}
\end{array}\right]
$$

Table 1: The matrix $Q$.

To express it as a quadratic form, in principle we need to define the new variables $z_{i}^{3}, z_{i}^{2} z_{j}(i \neq j)$ and $z_{i} z_{j} z_{k}(i \neq j \neq k)$. There are $n, n(n-1)$, and $\left(\begin{array}{l}n \\ 3\end{array}\right)$ different variables corresponding to each type. A particularly convenient ordering for the variables is the following:

$$
\begin{aligned}
\mathbf{Z}= & {\left[z_{1} z_{1}^{2}, z_{1} z_{2}^{2}, \ldots, z_{1} z_{n}^{2}, z_{2} z_{1}^{2}, z_{2} z_{2}^{2}, \ldots, z_{2} z_{n}^{2}, \ldots\right.} \\
& \left.z_{n} z_{1}^{2}, z_{n} z_{2}^{2}, \ldots, z_{n} z_{n}^{2}, z_{1} z_{2} z_{3}, \ldots, z_{n-2} z_{n-1} z_{n}\right]
\end{aligned}
$$

As in the case of the quartic form described earlier, without loss of generality it is always possible to choose some multipliers to be identically zero. This induces a block diagonal structure in the matrix $Q$, simplifying the final conditions, given in the theorem below.

Theorem 1 Consider the system of LMIs given by:

$$
\begin{aligned}
M-\Lambda^{i} & \geq 0, & & i=1, \ldots, n \\
\Lambda_{i i}^{i} & =0, & & i=1, \ldots, n \\
\Lambda_{j j}^{i}+\Lambda_{j i}^{j}+\Lambda_{i j}^{j} & =0, & & i \neq j \\
\Lambda_{j k}^{i}+\Lambda_{k i}^{j}+\Lambda_{i j}^{k} & \geq 0, & & i \neq j \neq k
\end{aligned}
$$

where the $n$ matrices $\Lambda^{i} \in \mathbb{R}^{n \times n}$ are symmetric $\left(\Lambda_{j k}^{i}=\right.$ $\left.\Lambda_{k j}^{i}\right)$. If there exists a feasible solution, then $M$ is copositive. Furthermore, this test is at least as powerful as condition (3).

Proof: As explained earlier, the feasibility of the LMIs is equivalent to the existence of a sum of squares decomposition for $P_{1}(\mathbf{z})$, and establishes its nonnegativity. This in turn implies the nonnegativity of $P(z)$, and therefore the copositivity of $M$.

More explicitly, the nonnegativity of $P_{1}(\mathbf{z})$ follows immediately from the LMIs above, since

$$
\begin{gathered}
\sum_{j, k} m_{j k} x_{j} x_{k} \geq \sum_{j, k} \Lambda_{j k}^{i} x_{j} x_{k} \\
\Longrightarrow P_{1}(\mathbf{z})=\sum_{i, j, k} m_{j k} x_{i} x_{j} x_{k} \geq \sum_{i, j, k} \Lambda_{j k}^{i} x_{i} x_{j} x_{k},
\end{gathered}
$$

and the coefficients of this last form are nonnegative.
It is also possible to verify directly that if the LMIs (2) have a solution, then so does the system (4). Just let

$$
\Lambda_{i j}^{k}=\lambda_{i j}\left(1-\delta_{i k}-\delta_{j k}\right)
$$

where $\delta$ is the usual Kronecker symbol, and $\lambda_{i i}=$ 0 for all $i$. This is a consequence of the "nested" properties of the $P_{r}$-based tests.

As we have shown, this class of tests is at least as powerful as the standard condition (3). A question naturally arises: how conservative is this procedure? To this end, consider the following theorem of Pólya:

Theorem 2 [7, Section 2.24] Given a form $F\left(x_{1}, x_{2}, \ldots, x_{n}\right)$ strictly positive for $x_{i} \geq 0, \sum_{i} x_{i}>0$, then $F$ can be expressed as

$$
F=\frac{G}{H},
$$

where $G$ and $H$ are forms with positive coefficients. In particular, we can choose

$$
H=\left(x_{1}+x_{2}+\cdots+x_{n}\right)^{r}
$$

for a suitable $r$.

In the case of a strictly copositive $M$, applying the the orem to the associated positive definite form $P(\mathbf{z})$, it is clear then that there is a finite $r$ for which the condition based on $P_{r}$ is exact. However, the minimum $r$ cannot be chosen as a constant (uniformly over all the positive definite forms). In general (see [17]) the known lower bounds for $r$ usually involve a "condition number" for the form $P$ : the minimum $r$ grows as the form tends to degeneracy (nontrivial solutions). This is consistent with the computational complexity results mentioned in the introduction: if the value of $r$ was bounded above, then we could always produce a polynomial time certificate for copositivity (namely, the sum of squares decomposition of $P_{r}(\mathbf{z})$ ), contradicting NP $\neq$ co-NP. However, these bounds can also be conservative: even if $P$ has nontrivial zeros, it might be possible to prove copositivity with a small value of $r$, as the examples we present show. 


\section{Examples}

As a confirmation that the proposed technique can actually be stronger than the standard relaxations, we will consider some particular examples from previous works. A future version of the paper will include examples from practical applications, such as the ones mentioned in the introduction.

Consider the quadratic form associated with the matrix $J$ below.

$$
J=\left[\begin{array}{rrrrr}
1 & -1 & 1 & 1 & -1 \\
-1 & 1 & -1 & 1 & 1 \\
1 & -1 & 1 & -1 & 1 \\
1 & 1 & -1 & 1 & -1 \\
-1 & 1 & 1 & -1 & 1
\end{array}\right]
$$

This form, originally introduced by A. Horn, appeared previously in $[4,15]$. It has been noted in $[4$, note added in proof] that it is copositive, even though it does not satisfy the condition (3).

Nevertheless, it is still possible to prove its copositiveness by the method presented in this paper. For the numerical implementation of the presented procedure, we used the semidefinite programming solver SeDuMi $[20]$. Let $\mathbf{x}:=\left[x_{1}, x_{2}, x_{3}, x_{4}, x_{5}\right]^{T}$. Taking $r=1$, after solving the corresponding LMIs we obtain the decomposition:

$$
\begin{aligned}
&\left(\mathbf{x}^{T} J \mathbf{x}\right)\left(x_{1}+x_{2}+\right.\left.x_{3}+x_{4}+x_{5}\right)= \\
& x_{1}\left(x_{1}-x_{2}+x_{3}+x_{4}-x_{5}\right)^{2}+ \\
& x_{2}\left(x_{2}-x_{3}+x_{4}+x_{5}-x_{1}\right)^{2}+ \\
& x_{3}\left(x_{3}-x_{4}+x_{5}+x_{1}-x_{2}\right)^{2}+ \\
& x_{4}\left(x_{4}-x_{5}+x_{1}+x_{2}-x_{3}\right)^{2}+ \\
& x_{5}\left(x_{5}-x_{1}+x_{2}+x_{3}-x_{4}\right)^{2}+ \\
&- \\
& 4\left(x_{1} x_{2} x_{4}+x_{2} x_{3} x_{5}+x_{3} x_{4} x_{1}+x_{4} x_{5} x_{2}+x_{5} x_{1} x_{3}\right)
\end{aligned}
$$

from where copositivity of $J$ follows immediately.

This example can be generalized to a family of copositive forms, with interesting theoretical properties. Consider the following cyclic quadratic form in $n=3 m+2$ variables $(m \geq 1)$, analyzed in [1]:

$$
B(\mathbf{x}):=\left(\sum_{i=1}^{3 m+2} x_{i}\right)^{2}-2 \sum_{i=1}^{3 m+2} x_{i} \sum_{j=0}^{m} x_{i+3 j+1}
$$

where $x_{r+n}=x_{r}$. It is clear that the Horn form presented above corresponds to the special case $m=1$. It has been shown in [1] that this is an extreme copositive form. Therefore, since $B(\mathbf{x})$ is neither componentwise nonnegative nor positive semidefinite, it cannot satisfy condition (3). Generalizing the decomposition above, we have the following theorem:

Theorem 3 Let $B(\mathbf{x})$ be as in equation (5). Then, it has the decomposition:

$$
\begin{aligned}
B(\mathbf{x}) \sum_{i=1}^{n} x_{i}= & \sum_{i=1}^{n} x_{i}\left(\sum_{j=1}^{n} x_{j}-2 \sum_{j=0}^{m} x_{i+3 j+1}\right)^{2}+ \\
& \left.+4 \sum_{i=1}^{n} x_{i} \sum_{k=1}^{m} x_{i+3 k-2}\left(\sum_{j=k}^{m} x_{i+3 j}\right) 6\right)
\end{aligned}
$$

Proof: For notational simplicity, let $s_{i}(\mathbf{x}) \quad:=$ $\sum_{j=0}^{m} x_{i+3 j+1}$. Let $L(\mathbf{x})$ be the left-hand side of (6). Then,

$$
L(\mathbf{x})=\sum_{i=1}^{n} \sum_{j=1}^{n} \sum_{k=1}^{n} x_{i} x_{j} x_{k}-2 \sum_{i=1}^{n} \sum_{k=1}^{n} x_{i} x_{k} s_{i}(\mathbf{x})
$$

The first term in the right-hand size of (6) can be written as:

$$
\begin{aligned}
R_{1}(\mathbf{x})= & \sum_{i=1}^{n} x_{i}\left(\sum_{j=1}^{n} x_{j}-2 s_{i}(\mathbf{x})\right)^{2} \\
= & \sum_{i=1}^{n} \sum_{j=1}^{n} \sum_{k=1}^{n} x_{i} x_{j} x_{k}-4 \sum_{i=1}^{n} \sum_{j=1}^{n} x_{i} x_{j} s_{i}(\mathbf{x}) \\
& +4 \sum_{i=1}^{n} x_{i} s_{i}^{2}(\mathbf{x})
\end{aligned}
$$

Subtracting, we obtain:

$$
\begin{aligned}
L(\mathbf{x})-R_{1}(\mathbf{x}) & =2 \sum_{i=1}^{n} \sum_{j=1}^{n} x_{i} x_{j} s_{i}(\mathbf{x})-4 \sum_{i=1}^{n} x_{i} s_{i}^{2}(\mathbf{x}) \\
& =2 \sum_{i=1}^{n} x_{i}\left(\sum_{j=1}^{n} x_{j}-2 s_{i}(\mathbf{x})\right) s_{i}(\mathbf{x})
\end{aligned}
$$

Expanding inside the sum, and cancelling identical terms corresponding to different values of $i$, after some manipulations we obtain the expression:

$$
4 \sum_{i=1}^{n} x_{i} \sum_{k=1}^{m} x_{i+3 k-2}\left(x_{i+3 k}+x_{i+3(k+1)}+\cdots+x_{i+3 m}\right),
$$

from where the result follows.

\section{Conclusions}

A new SDP-based procedure for checking copositivity of a matrix was developed. It is stronger than the standard sufficient condition (equation (3)), as was shown with the Horn form example. An important advantage is that it can be applied to matrices with unknown coefficients, if the dependence is affine. This important property can be exploited [12] in the formulation of higher-order SDP relaxations for quadratic programs. 
The procedure raises several interesting questions. For example, we know that the relaxation presented in Theorem 1 cannot be exact, since it runs in polynomial time. What is the minimum order $n$ of a counterexample? For the case of the standard condition (3), the results in [4] show that it is exact for $n \leq 4$.

Furthermore, there seems to be important theoretical connections with "lift-and-project" methods $[9,18]$ for deriving valid inequalities in zero-one combinatorial optimization problems. These relationships, as well as the connections with the enhanced relaxations in [12], will be explored elsewhere.

Important practical issues to be addressed deal with the question of how to exploit the special structure present in the problem, in order to get more efficient algorithms.

Acknowledgments: The author would like to acknowledge the reviewers, in particular Reviewer \#3, for their careful reading and insightful comments.

\section{References}

[1] V. J. D. Baston. Extreme copositive quadratic forms. Acta Arithmetica, XV:319-327, 1969.

[2] M. D. Choi and T. Y. Lam. An old question of Hilbert. Queen's papers in pure and applied mathematics, 46:385-405, 1977.

[3] R. W. Cottle, J. S. Pang, and R. E. Stone. The linear complementarity problem. Academic Press, 1992.

[4] P. H. Diananda. On non-negative forms in real variables some or all of which are non-negative. Proceedings of the Cambridge Philosophical Society, 58:17-25, 1962.

[5] M. R. Garey and D. S. Johnson. Computers and Intractability: A guide to the theory of NPcompleteness. W. H. Freeman and Company, 1979.

[6] M. Hall and M. Newman. Copositive and completely copositive quadratic forms. Proc. Camb. Phil. Soc., 59:329-339, 1963.

[7] G. H. Hardy, J. E. Littlewood, and G. Pólya. Inequalities. Cambridge University Press, 1967.

[8] M. Johansson. Piecewise Linear Control Systems. $\mathrm{PhD}$ thesis, Lund Institute of Technology, 1999.

[9] L. Lovász and A. Schrijver. Cones of matrices and set-functions and 0-1 optimization. SIAM Journal on Optimization, 1(2):166-190, 1991.

[10] K. G. Murty and S. N. Kabadi. Some NPcomplete problems in quadratic and nonlinear programming. Mathematical Programming, 39:117-129, 1987.

[11] C. H. Papadimitriou and K. Steiglitz. Combinatorial Optimization: Algorithms and Complexity. Dover, 1998.
[12] P. A. Parrilo. Higher order semidefinite relaxations for quadratic programming. In Proceedings of the $3^{\text {th }}$ IEEE Conference on Decision and Control, 2000.

[13] P. A. Parrilo. On a decomposition for multivariable forms via LMI methods. In Proceedings of the American Control Conference, 2000.

[14] V. Powers and T. Wörmann. An algorithm for sums of squares of real polynomials. Journal of pure and applied algebra, 127:99-104, 1998.

[15] A. J. Quist, E. De Klerk, C. Roos, and T. Terlaky. Copositive relaxation for general quadratic programming. Optimization methods and software, 9:185-208, 1998.

[16] A. R. Rajwade. Squares, volume 171 of London Mathematical Society Lecture Note Series. Cambridge University Press, 1993.

[17] B. Reznick. Some concrete aspects of Hilbert's 17th problem. In Contemporary Mathematics, volume 253, pages 251-272. American Mathematical Society, 2000.

[18] H. D. Sherali and W. P. Adams. A hierarchy of relaxations between the continuous and convex hull representations for zero-one programming problems. SIAM J. Disc. Math., 3(3):411-430, 1990.

[19] N. Z. Shor. Class of global minimum bounds of polynomial functions. Cybernetics, 23(6):731-734, 1987. (Russian orig.: Kibernetika, No. 6, (1987), 9$11)$.

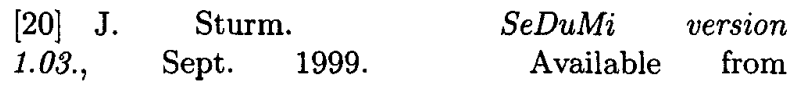
http://www . unimaas.nl/ sturm/sof tware/sedumi .html

[21] H. Väliaho. Criteria for copositive matrices. Linear Algebra and its applications, 81:19-34, 1986. 